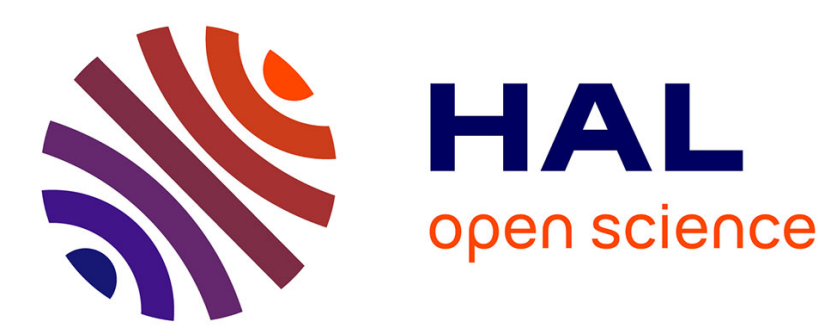

\title{
La révolution du livre de poche
}

Benoit Le Blanc

\section{To cite this version:}

Benoit Le Blanc. La révolution du livre de poche. Hermès, La Revue - Cognition, communication, politique, 2014, 70, pp.61-62. hal-02503637

\section{HAL Id: hal-02503637 \\ https://hal.science/hal-02503637}

Submitted on 10 Mar 2020

HAL is a multi-disciplinary open access archive for the deposit and dissemination of scientific research documents, whether they are published or not. The documents may come from teaching and research institutions in France or abroad, or from public or private research centers.
L'archive ouverte pluridisciplinaire HAL, est destinée au dépôt et à la diffusion de documents scientifiques de niveau recherche, publiés ou non, émanant des établissements d'enseignement et de recherche français ou étrangers, des laboratoires publics ou privés. 


\section{La révolution du livre de poche}

Avec l'avènement du livre de poche, le milieu du $\mathrm{XX}^{\mathrm{e}}$ siècle a été marqué par une véritable révolution, touchant à la fois la diffusion des savoirs et l'accès à la culture, proposant ni plus ni moins qu'une démocratisation de la littérature. Il ne s'agit pourtant pas d'une innovation à proprement parler, puisque deux générations précédentes sont identifiables: la première au $\mathrm{XIX}^{\mathrm{e}}$ siècle, avec des éditions dans un nouveau format compact (environ $11 \mathrm{~cm}$ sur $18 \mathrm{~cm}$ ) ; la seconde au tournant du $\mathrm{XX}^{\mathrm{e}}$ siècle, avec plusieurs collections de romans populaires (Olivero, 2007). Il existe également des antécédents au livre de poche, comme en Allemagne et en Angleterre dans les années 1930, suivis aux États-Unis par les "pocket books».

Ces livres de poche s'imposent sur la base d'un mode de production à bas coût couplé à une distribution à très large échelle. Il s'ensuit un nouvel objet de consommation pas toujours bien compris au départ: il est simple de présentation, dans un format pratique pour pouvoir être à disposition dans la poche et donc être lu dans les transports; il est proposé en librairie sur des supports modernes et disponibles partout, puisque tiré en très grand nombre d'exemplaires; il n'est pas cher et, au risque de se retrouver encombré, on peut en acheter beaucoup sans se sentir obligé de les lire jusqu'au bout; il se déforme à la lecture et se détériore à la lumière, mais son prix autorise de pouvoir le jeter après usage.

En France, le phénomène est identifié à la seule collection de la Librairie générale française, proposée depuis 1953 sous le nom de «Livre de poche». Existent pourtant la collection "J'ai Lu», introduite par Flammarion en 1958, et surtout «Folio», créée par Gallimard en 1972, qui retira alors au «Livre de poche» ses auteurs. Le livre de poche partage ainsi avec quelques autres fabricants de stylo, d'essuie-tout, de surligneur, de réfrigérateur ou encore de petit papier repositionnable, le privilège de coller son nom de marque à celui d'un objet populaire. L'idée majeure de son concepteur, Henri Filipacchi, est d'éditer des auteurs déjà reconnus en leur appliquant les nouvelles règles du marché de la consommation. Il y a là une réelle volonté de structurer la production et la diffusion pour obtenir un faible coût et toucher un large public. Ce mouvement est à comprendre dans le contexte de l'édition musicale de l'époque où les disques d'une idole pouvaient très bien se vendre à plusieurs centaines de milliers d'exemplaires en un temps très court. Avec le livre de poche, la littérature rejoignait les biens de grande consommation comme les cosmétiques, la nourriture ou la quincaillerie.

\section{Une légitimité qui fait débat}

Les acteurs du moment avaient bien conscience de vivre quelque chose d'exceptionnel, un tournant, sans trop savoir quelles conséquences cela aurait: qui achèterait ces livres et pourquoi? Pour les amoureux des reliures dorées et des bibliothèques, le choc était d'autant plus important que la mention «texte intégral», sur la couverture des livres de poche, signifiait bien le détachement du contenu (la littérature) de son contenant (le livre). Hormis les livres classiques toujours dominants sur le marché, les lecteurs modernes pouvaient désormais s'orienter vers des livres-club (œuvres choisies, objet soigné) ou vers des livres de poche (large catalogue, objet consommable) (Bouvy 1963).

Une fois le phénomène installé, c'est sa légitimité qui a été débattue. Les tenants d'une littérature pour 
les érudits et les partisans d'une nouvelle conception de la culture se sont ainsi affrontés entre 1964 et 1965 par revues interposées (Legendre 2010). Pour le Mercure de France, l'opération qui consiste à donner un accès sans fournir réellement de mode d'emploi relève de la mystification; pour Les Temps modernes, le rêve d'universalité de la culture sera atteint par une diffusion de masse. D’ailleurs, pour illustrer l'année 1964, la page Facebook du «Livre de poche» (<www.facebook.com/livredepoche $>$ ) reprend deux documents d'époque de l'Institut national de l'audiovisuel: l'un où un jeune étudiant bourgeois se moque de cette nouvelle prétention intellectuelle, l'autre où Marcel Pagnol salue ce nouveau moyen de culture aussi puissant que la radio ou la télévision.

Aujourd'hui, le livre de poche est omniprésent et représente le quart des ventes de livres en France. Il ne s'agit plus seulement de la déclinaison d'une collection en poche, mais bien de l'impression en format réduit de tout ce qui peut faire livre ou s'écrit. De nouvelles règles d'édition se sont mises en place depuis les années 1990, et la collection "poche» est devenue un passage obligé dans la stratégie de tous les éditeurs. Publié un an et demi après le grand format, le poche offre une seconde vie au livre, en le proposant à un prix de l'ordre du tiers de la parution initiale.

Par la rotation des titres, directement liée à la durée de présentation des parutions, le livre de poche a permis le succès de styles littéraires comme le roman policier ou

\section{RÉFÉRENCES BIBLIOGR A PHIQUES}

Bouvy, M., «Le livre de poche en France», Bulletin des bibliothèques de France [en ligne], $\mathrm{n}^{\circ} 11,1963$. Disponible sur: <bbf.enssib.fr/consulter/bbf-1963-11-0413-001>, consulté le 04/09/2014.

LEGENDRE, B., "Les débuts de l'édition de poche en France: entre l'industrie et le social (1953-1970)», Mémoire du livre, Stu- le fantastique. Une nouvelle forme de livre de poche s'est même installée depuis une vingtaine d'années: celle du livre au prix d'un café. Mais s'agit-il encore d'un livre? Les puristes ne le qualifieront que de brochure ou de fascicule. Toujours est-il que la démocratisation de la lecture a bel et bien eu lieu et que le livre est devenu un objet commun.

Revenons alors sur les questions fondamentales posées avec l'apparition du livre de poche. Qu'en est-il de la réalité d'un nouveau public? Ne s'agit-il pas plutôt là d'un effet d'aubaine pour les lecteurs, pouvant ainsi acheter plus de titres, et pour les éditeurs, pouvant accroître leurs ventes? Le public s'est effectivement élargi, même si la grande démocratisation n'a pas vraiment eu lieu. Les œuvres littéraires sont maintenant disponibles «en texte intégral» pour les élèves pendant leur scolarité. De façon générale, les jeunes ont un bon accès aux livres et les lisent, pour peu que les histoires les intéressent. L'acte d'achat ne recouvre toujours pas celui de la lecture effective, et ceci repose la question de la définition de ce qu'est «lire». On ne peut aimer lire que lorsque l'on sait lire; l'acte de la lecture est inséparable des notions d'effort, de curiosité et de plaisir. On remarquera, non sans une certaine malice, que ces questions reprennent toute leur actualité en ce moment, avec l'introduction des liseuses et de la lecture sur écran!

Benoît Le Blanc

École nationale supérieure de cognitique, Bordeaux-INP

dies in Book Culture [en ligne], vol. 2, $\mathrm{n}^{\circ} 1,2010$. Disponible sur: <www.erudit.org/revue/memoires/2010/v2/n1/045320ar.html>, consulté le 04/09/2014.

Olivero, I., "Le livre de poche: cinquante ans de succès », in Mollier, J.-Y. (dir.), Où va le livre? édition 2007-2008, Paris, La Dispute, 2007, p. 205-226. 